\title{
USO DAS INFORMAÇÕES FINANCEIRAS PARA O GERENCIAMENTO DE TÍTULOS DE RENDA VARIÁVEL: PERFIL DOS ANALISTAS DE INVESTIMENTO
}

\author{
S. V. RODRIGUES*, E. A. R. GARCIA, P. K. M. XIMENES e H. J. MOURA \\ Universidade de Fortaleza - UNIFOR \\ santiago.valcacer@gmail.com*
}

Artigo submetido em janeiro/2015 e aceito em maio/2015

DOI: 10.15628/holos.2015.2719

\section{RESUMO}

O estudo procura compreender o comportamento dos analistas de investimentos diante de sua preferência no posicionamento das ações negociadas na bolsa de valores brasileira (BM\&FBOVESPA) em 2012. A pesquisa tem uma abordagem de natureza preponderantemente quantitativa, por meio do método AHP Standard e da aplicação de testes estatísticos de correlação. A amostra do estudo foi construída por analistas de investimentos credenciados pela Associação dos Analistas e Profissionais de Investimento do Mercado de Capitais APIMEC em Fortaleza/CE. A metodologia foi aplicada às 10 ações mais negociadas e fez uso de 10 indicadores financeiros e de mercado mais utilizados na análise de decisão de investimentos em ativos financeiros. Os resultados apontam para uma falta de unanimidade entre os profissionais no que se refere à composição de portfólios. Ademais, o grupo de profissionais não seguiu, em grande parte, a lógica financeira esperada diante das variáveis estudadas. A investigação apontou para um comportamento heterogêneo por parte daqueles que fazem recomendações de investimentos aos clientes, assim como detectou sérias inconsistências por parte dos profissionais da área.

PALAVRAS-CHAVE: Finanças Comportamental, Analytic Hierarchy Process, Renda Variável.

\section{USE OF FINANCIAL INFORMATION FOR MANAGEMENT OF STOCK SECURITIES: PROFILE OF INVESTMENT ANALYSTS}

\begin{abstract}
The study seeks to understand the behavior of investment analysts in view of your preference in allocation of shares traded on the Brazilian Stock Exchange (BM\&FBOVESPA) in 2012. The research has a predominantly quantitative nature approach, through the AHP Standard method and the application of statistical correlation tests. The study sample was built by investment analysts accredited by the Association of Investment Analysts and Professionals of the Capital Markets - APIMEC in Fortaleza / CE. The methodology was applied to the 10 most traded shares and made use
\end{abstract}

of 10 financial indicators and market more used in the analysis of investment decisions in financial assets. The results point to a lack of unanimity among professionals regarding the composition of portfolios. In addition, the group of professionals not followed, largely, the expected financial logic on the variables studied. The investigation pointed to a mixed trend on the part of those who make investment recommendations to clients, as well as serious inconsistencies detected by professionals.

KEYWORDS: Behavioral Finance, Analytic Hierarchy Process, Stock Securities. 


\section{INTRODUÇÃO}

Uma das mais relevantes funções dos profissionais da área de investimento em títulos de renda variável é a de orientar seus clientes na formação de carteiras que se conformem aos respectivos perfis de risco/retorno (SPEIDELL, 2009). Independente da orientação "top down" ou "down up" o analista depende de informações econômicas e financeiras das empresas que atualmente são disponibilizadas de forma online por diferentes empresas, como: Sistema Economática, Bloomberg, Dun \& Bradstreet, entre outras, além da própria BM\&F Bovespa (PINHEIRO, 2009).

A operação em bolsa exige ampla análise tanto das variáveis macroeconômicas relacionadas ao desempenho da empresa, como as voltadas ao setor onde a mesma se insere. Um dos métodos amplamente utilizado baseia-se no uso de índices bursáteis, cujo propósito é o de avaliar se uma empresa específica está cara ou barata em termos relativos de mercado (PINHEIRO, 2009).

É esperado, entretanto, que diferentes especialistas se utilizem de distintos grupos de medidas que lhes sejam mais relevantes como suporte para prever o comportamento dos ativos financeiros. Observa-se um conflito persistente entre os defensores de que as melhores decisões de investimento baseiam-se em padrões do passado, com os que acreditam deverem ser as mesmas baseadas em níveis mais subjetivos sobre o futuro incerto (BERNSTEIN, 1997; KLOTZLE et al., 2012). Tal expectativa motivou o presente estudo, onde se procura conhecer em que medida as informações objetivas disponíveis - sejam contábeis, financeiras ou econômicas - contribuem ao profissional da área, no momento em que ele toma a decisão de investimento em renda variável.

Nessa corrente, Moura, Nogueira e Capelo (2011) propõem uma metodologia para estabelecer o perfil risco de analistas de investimento e a sua quantificação mediante o emprego de medidas adotadas pela teoria da decisão. Por outro modo, Klotzle et al. (2012) alçam uma medida para o desempenho comportamental dos analistas com base na teoria do prospecto.

Parte do processo da tomada da decisão de investimento é explicada pela Hipótese de Eficiência dos Mercados (HEM), proposta por Fama (1970), o qual afirma que o preço de um ativo reflete consistentemente todas as informações relevantes disponíveis. De acordo com a HEM, não há a possibilidade de se obter lucros anormais em mercados eficientes. Com efeito, segundo as três formas de eficiência propostas, nenhum investidor conseguiria ganhos em seus lucros de maneira anômala a partir exclusivamente dos preços históricos (forma "fraca"), apenas por meio de informações publicadas disponíveis (forma "semiforte"), bem como mediante a obtenção de informações privilegiadas (forma "forte").

Talvez por conta de algumas evidências empíricas favoráveis às hipóteses "fraca" e "semiforte, muitos profissionais de investimento fazem uso de informações financeiras ao selecionar títulos para recomendar aos seus clientes, já que se trata, em suma, de um processo de previsão futura baseado nas informações disponíveis. A propósito, trabalhos como os de Catapan e Catapan (2006) indicaram não haver relação significativa entre EBIT, retorno sobre patrimônio (RSPL) e preço das ações em empresas do setor elétrico. Por sua vez, o trabalho de Okimura e Sousa (2004) demonstra que a relação entre o preço da ação é maior quando 
correlacionada com o EVA do que em relação ao lucro líquido, embora não se configurem altas medidas de correlação. Conforme bem colocado por Eid Jr. e Pereira (2002), determinar parâmetros que reflitam adequadamente o desempenho das empresas é ainda um desafio para a teoria das finanças em razão, sobretudo, da existência de inúmeras variáveis inter-relacionadas que interferem substancialmente no movimento do mercado bursátil.

Por outro lado, a Teoria das Finanças Comportamentais (TFC) surge como crítica aos modelos tradicionais de tomada de decisão, ao buscar a compreensão da influência dos aspectos psicológicos no processo decisório dos agentes econômicos no mercado financeiro, uma vez que grande parte do pensamento humano baseia-se em histórias e justificativas subjetivas em contraposição a dados puramente quantitativos (BASU, HAJ, TCHALIAN, 2008; KIMURA, BASSO, KRAUTER, 2006; SPEIDELL, 2009; SUBRAHMANYAM, 2008).

Segundo os princípios da TFC, a racionalidade financeira é afetada pela influência de emoções e erros cognitivos, podendo gerar diferentes soluções para um mesmo problema, dependendo de como o mesmo foi abordado (MUSSA et al., 2008; SILVA et al., 2008; SUBRAHMANYAM, 2008). Ao introduzir por meio de estudos comportamentais a irracionalidade humana nas decisões e utilizar conceitos de outras áreas do conhecimento para dar suporte às premissas, a TFC pode ser considerada como aprimoramento da moderna teoria de finanças, esta última com alicerces construídos sob a racionalidade do agente econômico (KLOTZLE et al., 2012; MUSSA et al., 2008; ROGERS, SECURATO, RIBEIRO, 2007).

Diante do exposto, a questão norteadora do trabalho foi: "Até que ponto as informações financeiras históricas são úteis para os analistas de investimentos fazerem as suas recomendações?" Assim, o objetivo foi o de comparar os resultados das decisões tomadas pelos profissionais de investimentos em duas situações distintas:

(a) utilizando-se apenas de informações de natureza objetiva, quais sejam aquelas de natureza contábil, financeira e de mercado disponíveis no momento da tomada da decisão; e

(b) fazendo uso de qualquer outro tipo de informação (mesmo as anteriormente citadas), incluindo seu feeling e quaisquer outras percepções subjetivas.

A comparação dos dois experimentos possibilitará atender aos objetivos específicos seguintes:

quantificar o percentual de contribuição dos resultados baseados em medida objetiva (a) em relação aos obtidos por (b);

estabelecer comparativo entre os níveis de risco e retorno resultantes das duas abordagens, possibilitando caracterizar quantitativamente o perfil do decisor.

Como será detalhado na metodologia, o experimento de tomada de decisão se baseará na ferramenta AHP (Analytic Hierarchy Process), enquanto a formação do perfil do profissional de investimento fará uso do conceito de funções de utilidade.

É notório que o movimento nos preços dos ativos negociados em mercados financeiros tem retido a curiosidade tanto dos investidores quanto dos acadêmicos e vem se mostrado um importante campo de pesquisa há muitas décadas. Pode-se ainda observar que o mercado financeiro brasileiro passa atualmente por transformações profundas, envolvendo, principalmente, decisões de investimento no ambiente empresarial, o que tem levantado 
questionamentos acerca da aplicabilidade dos fundamentos relativos à teoria de finanças, questão extensivamente tratada no Brasil, dentre outros, por Kimura et al., (2006). Por sua vez, a crescente utilização da possibilidade de realizar investimentos diretos no Brasil, pelo sistema de Home Brokers, contribui para justificar a relevância do presente trabalho.

\section{RACIONALIDADE DO AGENTE ECONÔMICO}

Os principais fundamentos relacionados à racionalidade do investidor derivam da Moderna Teoria de Finanças, especificamente do arcabouço teórico proposto por Markowitz $(1952,1959)$ com a Teoria de Portfólio, do Modelo de Precificação de Ativos Financeiros (CAPM), proposto por Sharpe (1963), Lintner (1965) e Mossin (1966) e por último, da Hipótese de Eficiência de Mercados (HEM), elaborada por Fama (1970).

De acordo com os pressupostos da Moderna Teoria de Finanças, os agentes financeiros agem buscando sempre a maximização da riqueza e sua satisfação. Mais precisamente, o que se revela a partir das escolhas dos investidores relaciona-se às respectivas funções de utilidade e preferências com relação ao risco e ao retorno (PANDELO JR., 2010). Utilizando, porém a medida preconizada pelas Finanças Comportamentais (FC), a avaliação do risco passa a ser considerada em medições de perdas e ganhos. Neste caso, seria considerado racional o fato de os agentes apresentarem propensão a riscos no que se refere à perda e aversão relativamente aos ganhos (ROGERS et al., 2007).

As pesquisas em FC representam um campo ainda em franco desenvolvimento. Em grande parte, os estudos realizados sobre o perfil dos profissionais em finanças estão centrados na racionalidade de suas decisões. Contudo, essa racionalidade pode ser questionada a partir da influência de emoções e erros, fazendo com que as soluções para um mesmo problema sejam geradas por várias formas, dependendo de como o problema foi analisado (MUSSA et al., 2008).

Para Yoshinaga, Oliveira, Silveira e Barros (2008), a teoria das finanças até então adotada apresenta fragilidades no que se refere à sua validação empírica, pelo simples fato das pessoas não agirem sempre de forma racional. A partir desta constatação, as finanças comportamentais procuram incorporar as influências dos aspectos psicológicos inerentes ao comportamento dos agentes no processo decisório relacionado ao mercado financeiro, passando a validar os resultados empíricos observados (PIMENTA; RIBEIRO, 2010).

Os desvios do comportamento racional são inerentes à natureza humana, devendo ser incorporados à análise econômica do agente decisor, já que não podem ser descritos pelas condições de equilíbrio usuais, tendo em vista que os agentes financeiros tomam decisões muitas vezes incompatíveis com atitudes baseadas em expectativas racionais. De fato, os diversos aspectos psicológicos podem influenciar o comportamento humano, distorcendo a percepção dos fatos. É o que leva, segundo Kimura et al., (2006), a uma decisão baseada em julgamentos individuais, na qual a racionalidade imposta pela teoria da utilidade esperada pode não ser obedecida.

Já de acordo com a Prospect Theory (KAHNEMAN; TVERSKY, 1979; KLOTZLE et al., 2012), o indivíduo se caracteriza como avesso ao risco para ganhos, mas predisposto ao risco para perdas. Logo, este indivíduo optaria pela certeza de um ganho, em detrimento de algum risco cujo retorno esperado fosse o mesmo (CASTRO JR.; FAMÁ, 2002). Tal postura decerto afetaria o seu 
processo de tomada de decisão, impactando consequentemente os resultados financeiros realizados.

\section{TOMADA DE DECISÃO APOIADA POR ANALYTIC HIERARCHY PROCESS}

Frequentemente, as pessoas se deparam com a necessidade de tomada de decisões complexas. Para tomar uma decisão, de acordo com Saaty (2008), é preciso conhecer o problema, necessidade, finalidade, critérios e subcritérios, grupos afetados e as alternativas de ação a tomar. Goodwin e Wright (2004) argumentam que análise é a palavra-chave para a solução de problemas complexos, destacando que tais problemas envolvem múltiplos objetivos, incerteza, estrutura complexa e múltiplos stakeholders. Estes autores apresentam diversas técnicas utilizadas para a análise de decisões envolvendo múltiplos objetivos, como heurística, árvores de decisão, diagrama de influência, funções utilidade, SMART e AHP.

Para os autores, a heurística é utilizada em situações nas quais as pessoas encaram decisões sem suporte e estruturas de análise tendo como fatores essenciais: (i) o tempo disponível; (ii) o esforço envolvido; (iii) o conhecimento sobre o ambiente; (iv) a necessidade de justificar ou não a escolha a outras pessoas; e (v) o desejo de minimizar conflitos. Por sua vez, árvores de decisão podem ser empregadas na análise em etapas, representando uma estrutura analítica como suporte aos problemas de análise de decisão envolvendo incerteza. Estreitamente relacionado a árvores de decisão, os diagramas de influência resumem as dependências entre os eventos e as ações dentro de uma decisão.

Saaty (2008) apresentou a técnica AHP como abordagem voltada a problemas que envolvem incerteza. As principais etapas da ferramenta são: (i) definir a hierarquia da decisão; (ii) fazer comparações aos pares de atributos e alternativas; (iii) transformar as comparações em pesos e checar a consistência das comparações do tomador de decisão; (iv) usar os pesos para obter escores para as diferentes opções, tomando uma decisão provisória; e (v) realizar análise de sensibilidade e tomar a decisão definitiva.

A primeira etapa do AHP inclui os cursos de ação alternativos no nível hierárquico mais baixo da árvore de valores. O objetivo geral da tomada de decisão é colocado no topo, enquanto os atributos gerais associados ao objetivo são postos no nível imediatamente abaixo. Esses atributos podem ser segmentados e incorporar mais detalhes no próximo nível. Esse processo continua até o critério essencial para a tomada de decisão ser especificado. Finalmente, os cursos de ação são adicionados e a árvore de valor fica completa (GOODWIN; WRIGHT, 2004).

$\mathrm{Na}$ segunda etapa, para cada divisão na hierarquia, a importância do atributo é comparada com todos os outros atributos descendentes de um mesmo nível superior da árvore de valores. A escala de valores recomendada por Saaty (2008) vai de um (igualmente importante) a nove (extremamente mais importante). Cada conjunto de comparações pode ser representado por uma matriz, cujos valores indicam o quanto as linhas são mais importantes em relação às colunas. Números fracionários indicam que o atributo da coluna é mais importante. O processo é repetido até incluir as preferências relativas aos cursos de ação em relação a cada nível hierárquico mais baixo.

A terceira etapa converte os dados de cada tabela em um conjunto de pesos, automaticamente normalizados em soma um. Tal procedimento é repetido para cada grupo de 
variáveis consideradas, até que seja atingido o nível hierárquico mais baixo. Junto com os pesos, o AHP também calcula um índice de inconsistência, com a finalidade de alertar o tomador de decisão sobre qualquer inconsistência em relação às comparações que foram feitas. Um índice zero representa perfeita consistência do tomador de decisão.

Na quarta etapa, há a combinação de pesos para uma tomada de decisão provisória. Os escores para cada curso de ação são calculados pela soma dos produtos de todos os pesos dos atributos gerais ao curso de ação específico, devendo ser selecionado provisoriamente o curso de ação de maior escore. Finalmente, a última etapa é destinada à realização de análise de sensibilidade, pois é importante examinar quanto o curso de ação preferido é sensível a mudanças no julgamento feito pelo tomador de decisão.

Dentre as forças da ferramenta AHP, destacam-se: (i) necessidade de estruturar formalmente o problema; (ii) simplicidade da comparação aos pares; (iii) possibilidade de checagem das redundâncias; e (iv) versatilidade. As críticas apresentadas são: (i) conversão de comparações verbais em escala numérica; (ii) problemas com a escala de um a nove; (iii) aderência das respostas às questões; e (iv) o número de comparações requerido pode ser grande.

Como exemplos de uso do AHP, Frezatti et al., (2007) empregaram a técnica para investigar o relacionamento entre perfis da contabilidade gerencial e de planejamento de empresas. Gazzaneo (2008) utilizou a ferramenta para pré-seleção de ativos em uma tomada de decisão de investimentos. Oyatoye, Okpokpo e Adekoya (2010) o aplicaram na seleção de portfólio de investimento no setor bancário. Machado, Gomes e Chauvel (2008) propuseram o uso do AHP como apoio à decisão da análise de estratégias de marketing. Lee e Kozar (2006) aplicaram a abordagem para analisar o papel de um website no sucesso do comércio eletrônico. Moura et al., (2011) utilizaram o AHP como ferramenta para determinar os principais indicadores usados por profissionais de investimentos.

\title{
4 FUNÇÃO DE UTILIDADE
}

De acordo com Ragsdale (2004, p. 798),

\begin{abstract}
a teoria da utilidade assume que cada tomador de decisão possui uma função utilidade que traduz cada possível retorno de um problema em uma medida não monetária conhecida como utilidade. A utilidade de um retorno representa o valor associado a um determinado resultado de uma alternativa de decisão feita pelo tomador.
\end{abstract}

Uma hipótese fundamental que orienta a análise do comportamento do tomador de decisão é a racionalidade, i.e., assume-se que ele é capaz de avaliar as diferentes alternativas que Ihes são postas e escolher aquele que lhe garante o maior nível de satisfação ou utilidade possível (VARIAN, 1992). Vale ressaltar, entretanto, que essa hipótese vem sendo disputada pela teoria das finanças comportamentais que, através de experimentos e análises psicológicas, coletou evidências de que nem sempre os agentes são tão racionais assim quando encaram decisões arriscadas em um contexto de assimetria de informação (MOSCA, 2009).

Mais especificamente, dependendo de suas características e preferências, é possível perceber diferentes atitudes em relação a riscos e retornos por parte dos tomadores de decisão. 
Eles podem ser classificados como sendo neutros em relação ao risco, quando tomam decisões com base no valor médio esperado, i.e., quando a utilidade do retorno esperado é igual à utilidade esperada do retorno. Serão avessos ao risco, quando suas utilidades marginais são decrescentes em relação ao retorno, i.e., quando a utilidade do retorno esperado é maior que a utilidade esperada do retorno. E, finalmente, propensos ao risco, quando suas utilidades marginais crescem com o retorno, ou seja, quando a utilidade do retorno esperado é menor que a utilidade esperada do retorno (ALLEN et al., 2005; PETERSEN; LEWIS, 1999; RAGSDALE, 2004; VARIAN, 1992).

Uma das modalidades propostas para a criação das FUs para agentes avessos ao risco baseia-se no coeficiente de aversão ao risco, definido pela expressão $-U^{\prime \prime}(x) / U^{\prime}(x)$, que representa a 2a derivada da função utilidade, dividida pela 1a derivada, também conhecido como Coeficiente de Arrow-Pratt (MAS-COLLEL; WINSTON; GREEN, 1995). De fato, não é difícil construir tal tipo de função através de um modelo recursivo baseado em coeficientes angulares da forma ak = (1-CARk)ak-1, para o caso de funções lineares por partes. Entretanto, apesar da existência de métodos para a modelagem adequada de funções tal como o descrito acima, insiste-se aqui em um ponto crucial que é o de determinar cada um dos pontos da distribuição $\{x$, $\mathrm{U}(\mathrm{x})$ \}, obtida, como destacado acima, por métodos dissociados da realidade vivenciada pelo agente tomador da decisão.

Assim, uma das justificativas para o presente estudo é o propósito de determinar um método de atribuição de utilidades de forma intrínseca à atividade sobre a qual a FU será aplicada. Com efeito, de acordo com a descrição metodológica apresentada mais adiante, a FU será associada diretamente ao binômio risco x retorno adotado pelo profissional de investimentos.

\section{MÉTODOS}

A metodologia formulada visa à comparação das decisões subjetivas (tomadas pelo analista a partir da sua percepção) com aquelas baseadas em informações objetivas e explícitas disponíveis no momento, consistiu-se das seguintes etapas:

\subsection{Definição do Experimento}

O experimento consistiu em classificar 10 ações, dentre as mais negociadas do IBOVESPA, baseando-se na previsão do seu desempenho para um trimestre à frente. A data-base de realização do experimento foi 1\%/01/2013, quando ainda não se dispunha de informações para aquele ano, apesar de se ter acesso a uma base histórica relativa a períodos anteriores.

Dentre os 20 analistas de investimentos registrados na Associação dos Profissionais de Investimentos no Mercado de Capitais (APIMEC) na cidade de Fortaleza/CE no ano de 2012, selecionaram-se os $10 \mathrm{com}$ maior experiência profissional e atuação efetiva no mercado de capitais.

Solicitou-se a cada um deles que se colocasse no papel de assessor financeiro de um investidor conservador que na ocasião fosse detentor de portfólio com $50 \%$ aplicado em poupança, $30 \%$ em renda fixa e apenas $20 \%$ em ações. O profissional deveria classificar os 10 
títulos segundo uma escala de um a 10, onde um representava o melhor desempenho previsto para o próximo trimestre, enquanto 10 , o pior deles.

A classificação foi realizada de duas maneiras por cada analista:

Primeiramente, o profissional baseou-se exclusivamente na sua percepção, onde pôde fazer uso de todo o conhecimento. Nesta primeira situação, ele poderia levar em consideração aspectos de qualquer natureza (tais como feeling, projeção de cenários futuros, indicações internas e externas, etc.). Esta forma de classificação foi denominada Posicionamento Subjetivo (PS).

Em seguida, o mesmo analista utilizou-se apenas das informações disponíveis até 1\%/01/2013, envolvendo dados contábeis, financeiros e de mercado publicados pelo sistema Economática ${ }^{\circledR}$. Esta forma de classificação foi denominada Posicionamento Objetivo (PO).

Como forma de prover um maior grau de comparabilidade dos resultados encontrados, ambas alternativas fizeram uso de uma mesma métrica, baseada no processo AHP, por se tratar de técnica que possibilita uma estruturação formal do problema, demonstra simplicidade ao fazer comparações bivariadas, permitindo avaliar o grau de consistência das respostas. Na presente aplicação, a matriz de comparações binárias foi preenchida mediante o uso da tradicional convenção proposta por Saaty (2008): (1) Igualmente preferível; (3) Moderadamente preferível; (5) Fortemente preferível; (7) Muito fortemente preferível; (9) Extremamente preferível.

\subsection{Relação das ações utilizadas no experimento}

Dentre as ações que compunham o IBOVESPA em 31/12/2012, selecionaram-se as 10 mais negociadas, por se tratar de papéis que, em tese, deveriam ser amplamente conhecidos pelos profissionais da área de investimentos atuantes no mercado brasileiro. Tal definição teve o propósito de manter equivalência em relação ao nível de conhecimento dos participantes, de maneira que o experimento não fosse afetado por maiores diferenças na capacitação dos profissionais.

As ações consideradas foram:

Quadro 1: Amostra de 10 ações dentre as mais negociadas na BM\&F-Bovespa no último trimestre de 2012

\begin{tabular}{|l|c|c|c|c|c|c|c|c|c|c|}
\hline Código & PETR4 & VALE5 & ITUB4 & BBDC4 & BBAS3 & BVMF3 & PDGR3 & ITSA4 & OGXP3 & GGBR4 \\
\hline Empresa & Petrobrás & Vale & $\begin{array}{c}\text { Itaú } \\
\text { Unibanco }\end{array}$ & Bradesco & $\begin{array}{c}\text { Banco } \\
\text { Brasil }\end{array}$ & $\begin{array}{c}\text { BMF } \\
\text { Bovespa }\end{array}$ & $\begin{array}{c}\text { PDG } \\
\text { Realt }\end{array}$ & Itausa & $\begin{array}{c}\text { OGX } \\
\text { Petróleo }\end{array}$ & Gerdau \\
\hline Classe & PN & PNA & PN & PN & ON & ON & ON & PN & ON & PN \\
\hline
\end{tabular}

\subsection{Classificação baseada no posicionamento subjetivo (PS)}

O emprego do método AHP para a classificação das ações pressupõe a formação da árvore de valor e da matriz de comparações binárias mostradas a seguir: 


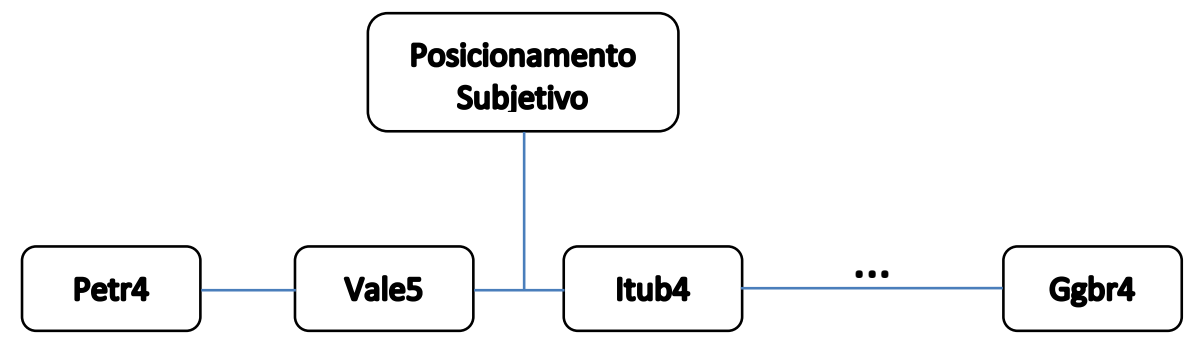

Figura 1: Árvore de Valor para AHP: Posicionamento Subjetivo Fonte: Elaborado pelos autores.

A partir da árvore de valor é traçada matriz de comparação binária conforme apresentado no quadro 2:

Quadro 2: Matriz de Comparações Binárias para Classificação do tipo PS

\begin{tabular}{|c|c|c|c|c|c|c|c|c|c|c|}
\cline { 2 - 12 } \multicolumn{1}{c|}{} & Petr4 & Vale5 & Itub4 & Bbdc4 & Bbas3 & Bvmf3 & Pdgr3 & Itsa4 & Ogxp3 & Ggbr4 \\
\hline Petr4 & 1 & $\mathrm{~W}_{12}$ & $\mathrm{~W}_{13}$ & $\mathrm{~W}_{14}$ & $\mathrm{~W}_{15}$ & $\mathrm{~W}_{16}$ & $\mathrm{~W}_{17}$ & $\mathrm{~W}_{18}$ & $\mathrm{~W}_{19}$ & $\mathrm{~W}_{110}$ \\
\hline Vale5 & $1 / \mathrm{W}_{12}$ & 1 & $\mathrm{~W}_{23}$ & $\mathrm{~W}_{24}$ & $\mathrm{~W}_{25}$ & $\mathrm{~W}_{26}$ & $\mathrm{~W}_{27}$ & $\mathrm{~W}_{28}$ & $\mathrm{~W}_{29}$ & $\mathrm{~W}_{210}$ \\
\hline Itub4 & $1 / \mathrm{W}_{13}$ & $1 / \mathrm{W}_{23}$ & 1 & $\mathrm{~W}_{34}$ & $\mathrm{~W}_{35}$ & $\mathrm{~W}_{36}$ & $\mathrm{~W}_{37}$ & $\mathrm{~W}_{38}$ & $\mathrm{~W}_{39}$ & $\mathrm{~W}_{310}$ \\
\hline \multicolumn{10}{|c}{} \\
\hline Ggbr4 & $1 / \mathrm{W}_{110}$ & $1 / \mathrm{W}_{210}$ & $1 / \mathrm{W}_{310}$ & $1 / \mathrm{W}_{410}$ & $1 / \mathrm{W}_{510}$ & $1 / \mathrm{W}_{610}$ & $1 / \mathrm{W}_{710}$ & $1 / \mathrm{W}_{810}$ & $1 / \mathrm{W}_{910}$ & 1 \\
\hline
\end{tabular}

Como pode ser observado, a matriz triangular inferior do quadro é calculada através do inverso da parte superior, representando o contrário das preferências atribuídas (SAATY, 2008).

Uma próxima etapa converte os dados em um conjunto de pesos, automaticamente normalizados e totalizando um.

Após totalizar as colunas e encontrar as proporções das linhas, os pesos de cada item da primeira coluna são encontrados mediante o cálculo das médias das linhas.

Junto com os pesos, o AHP também calcula um índice de inconsistência, com a finalidade de alertar o tomador de decisão sobre eventuais inconsistências quanto às relações de transitividade das respostas. Tal medida baseia-se em uma razão de consistência (RC) definida por Saaty (2008) como:

$$
\mathrm{RC}=\frac{\mathrm{IC}}{\mathrm{IA}}
$$

onde:

O numerador IC representa um índice de consistência, dado por $(\lambda \max -n) /(n-1)$, em que $\lambda$ max é o maior autovalor (eingenvalue) da matriz e $n$, o número de colunas (ou linhas). Por sua vez, o denominador IA consiste de um índice aleatório, definido para este caso particular por $1,99(n-2) / n$. Um índice de zero indica consistência perfeita no que tange às relações de transitividade das respostas.

Ao final desta etapa, é possível priorizar as 10 ações selecionadas, mediante a atribuição de pesos gerados pela técnica AHP, doravante denominados de Escores Subjetivos e representados pelo vetor $\{\mathrm{Si}\}$, onde $\mathrm{i}=1,2,3 \ldots 10$. 


\subsection{Classificação baseada no posicionamento objetivo (PO)}

Neste caso, o posicionamento das ações envolveu duas etapas:

- Classificação dos indicadores usados para classificar os papéis;

- Posicionamento dos próprios papéis, a partir da ponderação estabelecida acima.

De fato, apresentou-se inicialmente ao participante a relação de vários indicadores dentre os disponíveis no sistema Economática, para que ele os classificasse segundo o grau de importância. Em seguida as ações foram posicionadas segundo os pesos referentes à relevância de cada indicador.

Quadro 3: Os Indicadores Julgados Relevantes pelos Analistas

\begin{tabular}{|c|c|l|}
\hline \hline Representação & Indicador & \multicolumn{1}{c|}{ Descrição } \\
\hline \hline Ind 1 & Preço / Lucro & $\begin{array}{l}\text { Preço da Ação em 31 de dezembro de 2012, dividido pelo lucro líquido } \\
\text { do último trimestre de 2012. }\end{array}$ \\
\hline Ind 2 & Preço / Vendas & $\begin{array}{l}\text { Preço da Ação em 31 de dezembro de 2012, dividido pelo volume de } \\
\text { vendas do último trimestre de 2012. }\end{array}$ \\
\hline Ind 3 & Preço / EBITDA & $\begin{array}{l}\text { Preço da Ação em 31 de dezembro de 2012, dividido pelo EBITDA do } \\
\text { último trimestre de 2012. }\end{array}$ \\
\hline Ind 4 & Dividend Yeld & Rendimento dos Dividendos Anuais ao final do ano de 2012. \\
\hline Ind 5 & Enterprise Value / EBIT & $\begin{array}{l}\text { Valor da Empresa em 31 de dezembro de 2012, dividido pelo EBIT do } \\
\text { último trimestre de 2012. }\end{array}$ \\
\hline Ind 6 & Lucro Por Ação & $\begin{array}{l}\text { Lucro Líquido da Empresa no último trimestre de 2012, dividido pelo } \\
\text { número de ações. }\end{array}$ \\
\hline Ind 7 & Valor Patrimonial da Ação & $\begin{array}{l}\text { Valor Patrimonial da Empresa no último trimestre de 2012, dividido pelo } \\
\text { número de ações. }\end{array}$ \\
\hline Ind 8 & EBIT/Vendas & $\begin{array}{l}\text { EBIT da empresa dividido pelo volume de vendas, ambos do último } \\
\text { trimestre de 2012. }\end{array}$ \\
\hline Ind 9 & Retorno & $\begin{array}{l}\text { Retorno ajustado por proventos (inclusive dividendos) dos preços de } \\
\text { fechamento de 01 de outubro a 28 de dezembro de 2012 }\end{array}$ \\
\hline Ind 10 & Volatilidade & $\begin{array}{l}\text { Coeficiente de variação medido em base diária, do último trimestre de } \\
\text { 2012. }\end{array}$ \\
\hline \hline
\end{tabular}

Fonte: Elaborado pelos autores.

O procedimento implementado pelo processo AHP é sumarizado pela árvore de valor seguinte:

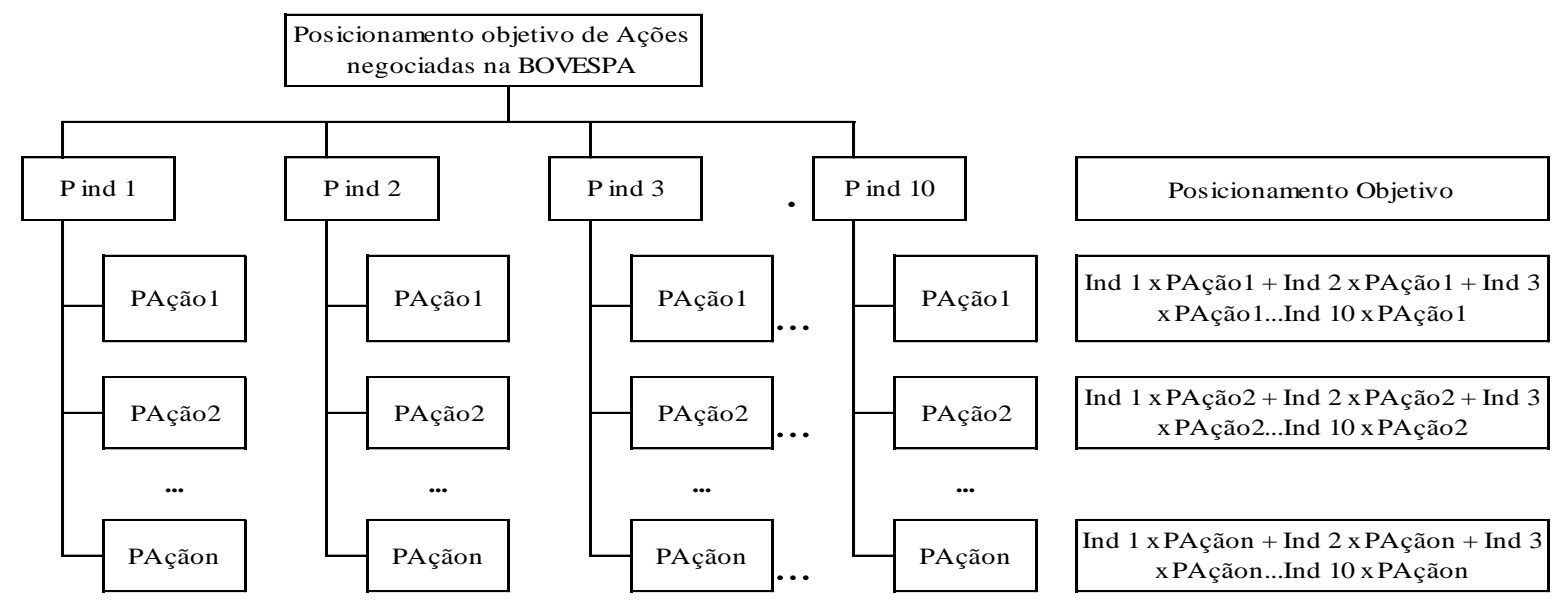

Figura 2: Árvore de valor para AHP: Posicionamento Objetivo Fonte: Elaborado pelos autores. 
De forma equivalente à etapa 4.3, foi necessário formar matriz de comparação binária, agora em duas etapas: uma para classificar os indicadores e outra para classificar as ações a partir da ponderação dos indicadores.

Os pesos associados aos indicadores foram representados pelo vetor $\{\mathrm{Wi}\}$, com $i=1,2,3 \ldots 10$, enquanto aqueles referentes às ações em cada indicador, pela matriz $\{A i, j\}$, onde ambos os índices variam de 1 a 10.

A partir daí foi possível obter os denominados Escores Objetivos (Oi), calculados como:

$$
\mathrm{O}_{\mathrm{i}}=\sum_{i, j} W_{j} \times A_{i, j}
$$

\subsection{Relacionamento entre PS, PO e Valores Reais}

As etapas anteriores possibilitaram montar o quadro abaixo, o qual se constitui na principal estrutura de dados utilizada na investigação:

\section{Quadro 3: Posicionamento Geral}

\begin{tabular}{|c|c|c|c|c|}
\hline Ação & $\begin{array}{c}\text { Escore } \\
\text { Subjetivo }\end{array}$ & Escore Objetivo & $\begin{array}{c}\text { Retorno } \\
\text { Esperado }\end{array}$ & Risco ${ }^{\text {(2) }}$ \\
\hline Petr4 & $\mathrm{S}_{1}$ & $\mathrm{O}_{1}$ & $\mathrm{E}_{1}$ & $\mathrm{R}_{1}$ \\
\hline Vale5 & $\mathrm{S}_{2}$ & $\mathrm{O}_{2}$ & $\mathrm{E}_{2}$ & $\mathrm{R}_{2}$ \\
\hline Itub4 & $\mathrm{S}_{3}$ & $\mathrm{O}_{3}$ & $\mathrm{E}_{3}$ & $\mathrm{R}_{3}$ \\
\hline \multicolumn{5}{|c|}{} \\
\hline Ggbr4 & $\mathrm{S}_{10}$ & $\mathrm{O}_{10}$ & $\mathrm{E}_{10}$ & $\mathrm{R}_{10}$ \\
\hline
\end{tabular}

Fonte: Elaborado pelos autores.

(1) Refere-se ao retorno esperado do 10 trimestre de 2013

(2) Refere-se ao coeficiente de variação do 1 을 trimestre de 2013

Enquanto a forma de obter as duas primeiras colunas já foi detalhada e os números resultantes referem-se a valores projetados em 31/12/2012 para o primeiro trimestre de 2013, as duas seguintes contêm os números efetivos observados no primeiro trimestre de 2013. Conforme pode ser observado, o posicionamento real dos títulos foi segmentado nas duas dimensões: retorno esperado e risco.

Com base nestes números, foi então possível encontrar o relacionamento entre todas as variáveis, para o qual se fez uso dos coeficientes de Pearson e Spearman para análise das correlações absoluta e serial, do coeficiente de determinação R2 para verificar o grau de explicação de $\{\mathrm{O} i\}$ sobre $\{\mathrm{Si}\}$ e da construção de um gráfico tridimensional para representar o perfil do analista, através de uma proxy de função de utilidade.

A seção seguinte apresenta os valores que foram obtidos, assim como a análise de tais resultados.

\section{RESULTADOS}

Segundo o que foi detalhada na metodologia, a aplicação do processo AHP gerou os valores abaixo, representativos do posicionamento das ações segundo as duas abordagens 
citadas. Embora tenha sido aplicada aos 10 profissionais selecionados, a Tabela 1 ilustra os resultados de apenas um deles:

Tabela 1 - Matriz de Resultados das Recomendações de um Analista

\begin{tabular}{|c|c|c|c|c|c|c|c|c|c|c|}
\hline Ação & Petr4 & Vale5 & Itub4 & Bbdc4 & Bbas3 & Bvmf3 & Pdgr3 & Itsa4 & Ogxp3 & Ggbr4 \\
\hline Petr4 & 1 & 0,200 & 0,333 & 0,333 & 0,333 & 3 & 1 & 0,143 & 1 & 0,200 \\
\hline Vale5 & 5 & 1 & 5 & 3 & 3 & 7 & 7 & 0,143 & 7 & 5 \\
\hline Itub4 & 3 & 0,200 & 1 & 3 & 7 & 5 & 7 & 0,333 & 7 & 5 \\
\hline \multicolumn{10}{|c|}{ Fonte: Elaborado pelos autores por meio das recomendações dos analistas. }
\end{tabular}

Após totalizar as colunas e encontrar as proporções das linhas, os posicionamentos subjetivos de cada analista são encontrados mediante o cálculo das médias das linhas, resultando em:

Tabela 2 - Posicionamento Subjetivo para o analista A1

\begin{tabular}{|l|c|c|c|c|c|c|c|c|c|c|}
\hline \hline Ação & Petr4 & Vale5 & Itub4 & Bbdc4 & Bbas3 & Bvmf3 & Pdgr3 & Itsa4 & Ogxp3 & Ggbr4 \\
\hline PS & 0,031 & 0,192 & 0,158 & 0,135 & 0,081 & 0,025 & 0,033 & 0,26 & 0,018 & 0,067 \\
\hline \multicolumn{8}{|c|}{ Fonte: } & Elaborado pelos autores por meio das recomendações dos analistas
\end{tabular}

Nesta ilustração, constata-se que este profissional atribuiu melhor classificação ao título Vale5, deixando Ogxp3 em último lugar.

Seguindo o que foi definido na metodologia, obtiveram-se os PS e PO para cada um dos 10 analistas componentes da amostra, possibilitando relacionar os valores obtidos da forma seguinte:

\subsection{Relação entre os Posicionamentos Subjetivo e Objetivo}

A tabela seguinte mostra os coeficientes de Correlação de Spearman para a amostra considerada:

Tabela 3 - Coeficientes de Correlação entre PS e PO

\begin{tabular}{c|c|c|c|c|c|c|c|c|c|c}
\hline \hline \multicolumn{1}{c|}{ Analistas } & A1 & A2 & A3 & A4 & A5 & A6 & A7 & A8 & A9 & A10 \\
\hline \hline Coeficiente de Correlação & 0,164 & 0,494 & 0,624 & 0,576 & 0,455 & 0,479 & $-0,697$ & 0,600 & 0,139 & 0,273 \\
\hline Sig. (2-tailed) & 0,651 & 0,147 & 0,054 & 0,082 & 0,187 & 0,162 & 0,025 & 0,067 & 0,701 & 0,446 \\
\hline \hline
\end{tabular}

Os números indicam que, à exceção de três analistas (A3, A7 e A8), as classificações das ações geradas pelas duas formas (PS e PO) não são significativas, levando a cogitar a princípio que a análise objetiva baseada nas informações existentes não se relaciona com a recomendação final do analista. Contudo, o fato dos coeficientes serem positivos aponta para uma mesma direção quanto ao uso das duas formas de posicionamento. Aspecto interessante foi observado no caso do analista A7, cujo posicionamento objetivo foi fortemente inverso ao subjetivo, indicando que a sua classificação das ações foi quase totalmente inversa ao recomendado pelas informações disponíveis.

O uso do coeficiente de determinação, baseado em regressão da forma $P S=f(P O)$, permitiu constatar que no caso dos mesmos profissionais citados acima, houve um único caso de contribuição significativa (a 5\%) dos indicadores objetivos, levando a um R2 de 0,41, indicando 
que $41 \%$ do posicionamento atribuído pelo analista se devem ao uso das informações objetivas. Contudo, pelo fato do coeficiente de regressão linear ser negativo, trata-se do mesmo caso citado anteriormente (analista A7), em que o conhecimento de PO contribui de forma inversa para o conhecimento de PS.

\subsection{Comparação das Classificações PS e PO com os Resultados Efetivos}

Deve ser lembrado que os profissionais foram solicitados a fazer suas classificações no final de 2012, quando obviamente ainda não se conhecia os resultados de 2013. Os resultados abaixo consistem nas comparações entre ambos os posicionamentos com os resultados efetivamente observados no primeiro trimestre de 2013 , possibilitando verificar qual das duas abordagens se mostrou mais próxima dos resultados reais.

Como detalhado na metodologia, os dados efetivos foram segmentados em retorno esperado e volatilidade, resultando na tabela seguinte:

Tabela 4 - Coeficientes de Correlação Ordinais (Spearman) entre as Formas de Posicionamento das Ações e os Retornos e Volatilidades Efetivos

\begin{tabular}{|c|c|c|c|c|c|c|c|c|c|c|}
\hline Analista & A1 & A2 & A3 & A4 & A5 & A6 & A7 & A8 & A9 & A10 \\
\hline \hline \multicolumn{10}{|c|}{ Retorno } \\
\hline PS & 0,60 & 0,82 & 0,16 & 0,54 & 0,33 & 0,20 & $-0,35$ & 0,87 & 0,50 & 0,33 \\
\hline Sig. & 0,07 & 0,00 & 0,65 & 0,11 & 0,35 & 0,58 & 0,33 & 0,00 & 0,14 & 0,35 \\
\hline PO & 0,54 & 0,55 & 0,55 & 0,54 & 0,44 & 0,48 & 0,54 & 0,47 & 0,53 & 0,58 \\
\hline Sig & 0,11 & 0,10 & 0,10 & 0,11 & 0,20 & 0,16 & 0,11 & 0,17 & 0,12 & 0,08 \\
\hline \multicolumn{10}{|c|}{ Risco } \\
\hline PS & $-0,76$ & $-0,94$ & $-0,44$ & $-0,88$ & $-0,12$ & $-0,27$ & 0,58 & $-0,77$ & $-0,61$ & $-0,16$ \\
\hline Sig. & 0,01 & 0,00 & 0,20 & 0,00 & 0,75 & 0,45 & 0,08 & 0,01 & 0,06 & 0,65 \\
\hline PO & $-0,53$ & $-0,54$ & $-0,54$ & $-0,62$ & $-0,52$ & $-0,48$ & 0,39 & $-0,62$ & $-0,47$ & $-0,55$ \\
\hline Sig. & 0,12 & 0,11 & 0,11 & 0,05 & 0,13 & 0,16 & 0,26 & 0,05 & 0,17 & 0,10 \\
\hline
\end{tabular}

Fonte: Elaborado pelos autores

A primeira parte da tabela mostra os coeficientes de correlação de Spearman entre os posicionamentos baseados em ambos os tipos de comportamento (PS e PO) com a classificação efetiva das ações baseada na rentabilidade observada no primeiro trimestre de 2013. Nota-se que apenas três dos profissionais (A1, A2 e A8) mostraram correlação PS x efetivo superior a PO $x$ efetivo, sendo que apenas duas foram significantes. A maior parte dos pesquisados apresentou resultados baseados em PO iguais ou superiores aos de PS sem haver, no entanto, significância estatística nos resultados. Os resultados indicam que a classificação dos papéis baseada em indicadores objetivos e disponíveis na data do experimento foi mais aderente às rentabilidades reais, do que aqueles guiados pelas percepções dos profissionais.

A segunda parte da tabela mostra correlações entre as classificações baseadas em PS e PO e aquelas baseadas na volatilidade e efetivamente observadas em 2013. Neste segundo caso, observa-se que praticamente todos os profissionais - à exceção de A7 - posicionaram os títulos de maneira inversa à proporcionada pela dimensão do risco, levando a conjecturar que os profissionais priorizam muito mais fortemente a rentabilidade dos títulos em detrimento do risco associado, como mostram as correlações negativas. Além disso,, observou-se que seis das correlações PS, quando consideradas em valor absoluto, são maiores do que as PO, revelando que também no caso da dimensão risco, as classificações subjetivas são mais negativamente correlacionadas com a realidade do que aquelas baseadas em índices e variáveis objetivas. 


\subsection{Visualização do Perfil do Profissional de Investimentos}

O gráfico tridimensional a seguir mostra a rentabilidade e o risco de cada uma das 10 ações consideradas, além da classificação proposta por quatro analistas e baseada no posicionamento subjetivo.

Observa-se que o Gráfico 1 representa uma ilustração do perfil gráfico do profissional e considerou apenas quatro analistas, a fim de não sobrecarregar a visualização. É possível observar, por exemplo, que enquanto os profissionais A1, A5 e A7 atribuíram ao título OGXP3 uma baixa classificação, o analista A7 o posicionou melhor, a despeito da sua baixa rentabilidade e alto risco demonstrado.
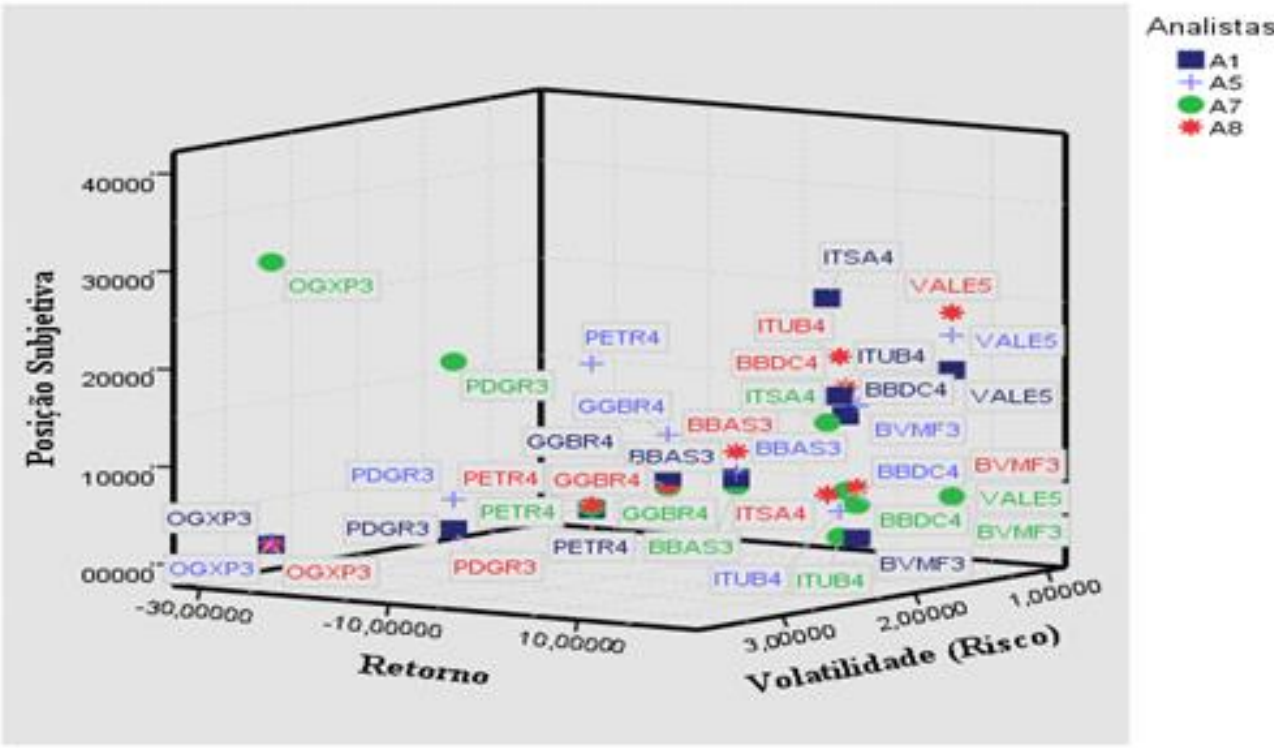

Figura 3: Posicionamento Subjetivo, Versus Retorno e Risco dos Analistas A1, A5, A7 e A8. Fonte: Elaborado pelos autores.

A figura demonstra a possibilidade de se atribuir perfis gráficos de retorno e risco aos profissionais de investimento, permitindo criar uma espécie de função de utilidade tridimensional de forma mais prática e diferente da usual, na qual se costuma indagar dos pesquisados a respeito de loterias baseadas em situações fictícias.

\section{CONCLUSÃO}

Os resultados apontaram para uma maior aderência das projeções baseadas em valores objetivos do que em percepções completas do analista, tanto sob a perspectiva de rentabilidade como na de risco. Contudo, há que se fazerem algumas ressalvas:

a) O final do ano de 2012 representou um ponto de inflexão importante no tocante à rentabilidade do mercado de capitais brasileiro, o que decerto impactou as projeções dos pesquisados.

b) A maior pretensão do trabalho foi mostrar a viabilidade de avaliar quantitativamente o comportamento dos profissionais de investimento, determinando inclusive uma função de utilidade para eles. De fato, os resultados encontrados, a despeito de serem 
provenientes de profissionais experientes do mercado de capitais brasileiro, são insuficientes para concluir definitivamente sobre as melhores formas de selecionar ações.

Não obstante, os resultados indicaram uma completa divergência de estratégias de investimento, mesmo quando aplicada sobre pequeno grupo de profissionais reconhecidos e qualificados, com nível de conhecimento técnico similar.

No caso particular deste experimento, observou-se que as informações contábeis e financeiras disponíveis no momento da tomada de decisão de investimentos mostraram-se relevantes para o processo, sobressaindo-se, em grande parte das vezes, ao conhecimento subjetivo do profissional.

Outro aspecto observado foi o fato da baixa importância dada ao risco dos papéis por parte dos profissionais. Tal aspecto se reveste de importância ao lembrar que no início do experimento foi informado que o cliente tinha natureza conservadora, sendo detentor de uma carteira com $50 \%$ aplicado em poupança, 30\% em renda fixa e apenas $20 \%$ em ações.

Finalmente, algo que se coaduna com os resultados encontrados por outras pesquisas sobre o tema, como por exemplo, a realizada por Moura et al. (2011), é a forte heterogeneidade das decisões de investimento dos profissionais da área. Tal fato decerto remete à intrigante questão: além do risco inerente aos próprios títulos de renda variável que pretende investir, é pertinente que o aplicador considere o risco do assessoramento que está recebendo?

Do ponto de vista da modelagem, este trabalho indica que é razoável supor que modelos bayesianos, em que probabilidades "a posteriori" dependam do perfil do consultor, se configurem em ferramentas mais completas e adequadas ao investidor do que os tradicionais instrumentos fornecidos pela teoria de finanças.

\section{REFERÊNCIAS}

1. ALLEN, W. B.; DOHERTY, N. A.; MANSFIELD, E.; WEIGELT, K. Managerial Economics: theory, applications, and cases. 6th ed. Nova York: W. W. Norton \& Company, 2005.

2. BASU, S.; RAJ, M.; TCHALIAN, H. A Comprehensive Study of Behavioral Finance. Journal of Financial Service Professionals, v. 62, n. 4, p. 51-62, 2008.

3. BERNSTEIN, P. L. Desafio aos Deuses: A Fascinante Historia do Risco. Rio de Janeiro: Elsevier, 1997.

4. CASTRO JR., F. H. F. DE; FAMÁ, R. As novas finanças e a teoria comportamental no contexto da tomada de decisão sobre investimentos. Caderno de Pesquisas em Administração, São Paulo, v. 09, n. 2, abril/junho, 2002.

5. CATAPAN, A.; CATAPAN, E. (2006). Rentabilidade e endividamento do setor elétrico brasileiro: um enfoque sobre sustentabilidade organizacional. ANAIS DO ENCONTRO NACIONAL DE ENGENHARIA DE PRODUÇÃO, Fortaleza, CE, Brasil, 26, Outubro, 2006.

6. EID JR, W.; PEREIRA, S. C. Medidas de criação de valor e retorno das ações. ANAIS DO ENCONTRO NACIONAL DA ASSOCIAÇÃO NACIONAL DE PÓS-GRADUAÇÃO E PESQUISA EM ADMINISTRAÇÃO - ENAPAD, Salvador, BA, Brasil, 26, setembro, 2002.

7. FAMA, E.F. Efficient Capital Markets: a review of theory and empirical work. Journal of 
Finance, Chicago: American Finance Association, p.383-417, maio, 1970.

8. FREZATTI, F.; GUERREIRO, R.; AGUIAR, A.; GOUVÊA, M. Análise do Relacionamento entre a Contabilidade Gerencial e o Processo de Planejamento das Organizações Brasileiras. Revista de Administração Contemporânea. 2 Edição Especial, p. 33-54, 2007.

9. GAZZANEO, B. Pré-seleção de ações para a construção de carteiras eficientes. (Dissertação de Mestrado) Faculdades IBMEC, Rio de Janeiro, RJ, Brasil, 2008.

10. GOODWIN, P.; WRIGHT, G. Decision Analysis for Management Judgment. 3. ed. West Sussex: John Wiley \& Sons Ltd, 2004.

11. KAHNEMAN, D.; TVERSKY, A. Prospect theory: an analysis of decision under risk. Econometrica, v. 47, n. 2, p. 263-291, 1979.

12. KIMURA, H.; BASSO, L. F. C.; KRAUTER, E. Paradoxos em finanças: teoria moderna versus finanças comportamentais. Revista de Administração de Empresas - ERA - FGV, São Paulo, v. 46, n 1, p. 41-58, jan/mar, 2006.

13. KLOTZLE, M. C.; GOMES, L. L.; BRANDÃO, L. E. T.; PINTO, A. C. F. Desenvolvimento de uma medida de desempenho comportamental. Revista Brasileira de Finanças, Rio de Janeiro, v. 10, n. 3, p. 395-416, 2012.

14. LEE, A.; KOZAR, K. Investigating the effect of website quality on e-business success: An analytic hierarchy process (AHP) approach. Decision Support Systems, v. 42, p.1383-1401, 2006.

15. LINTNER, J. Security prices, risk, and the maximal gains from diversification. Journal of Finance, v. 20, n. 4, p. 587-615, 1965.

16. MACHADO, E.; GOMES, L.; CHAUVEL, M. Avaliação de estratégias em marketing de serviços: Um enfoque multicritério. Revista de Administração Mackenzie, v. 4, n. 2, p. 61-85, 2008.

17. MARKOWITZ, H. M. Portfolio Selection. The Journal of Finance, Chicago: American Finance Association, v. 7, n. 1, p. 77-91, 1952.

18. _ (1959). Portfolio Selection. New York: John Wiley \& Sons.

19. MAS-COLELL, A.; WINSTON, M. D.; GREEN, J. R. Microeconomic theory. Oxford: Oxford University Press, 1995.

20. MOSCA, A. Finanças Comportamentais: gerencie suas emoções e alcance sucesso nos investimentos. Rio de Janeiro: Elsevier (Expo Money), 2009.

21. MOSSIN, J. Equilibrium in a Capital Asset Market. Econometrica, v. 34, n. 4, Oct., pp.768-783, 1966.

22. MOURA, H. J.; NOGUEIRA, C. A. G.; CAPELO, E. JR.; BORGES, J. F. Proposta para determinação do perfil de profissionais de investimento por meio de funções de utilidade. ANAIS DO XXXV ENCONTRO DA ANPAD, Rio de Janeiro, 2011.

23. MUSSA, A.; YANG, E.; TROVÃO, R.; FAMÁ, R. Hipótese de Mercado Eficiente e Finanças Comportamentais: As discussões persistem. Revista FACEF PESQUISA - v.11, n.1, 2008.

24. OKIMURA, R. T.; SOUSA, A. F. O valor adicionado possui maior correlação com o retorno das ações do que o lucro líquido no Brasil. ANAIS DO ENCONTRO NACIONAL DA ASSOCIAÇÃO NACIONAL DE PÓS-GRADUAÇÃO E PESQUISA EM ADMINISTRAÇÃO, Curitiba, PR, Brasil, 28, 
Setembro, 2004.

25. OYATOYE, E.; OKPOKPO, G.; ADEKOYA, G. An application of analytic hierarchy process (AHP) to investment portfolio selection in the banking sector of the Nigerian capital market. Journal of Economics and International Finance, v. 2, n. 12, p. 321-335, 2010.

26. PANDELO JR, D. R. Análise do Perfil do Investidor com Base em Análise de suas Percepções Subjetivas de Risco e Retorno. Revista Cesumar - Ciências Humanas e Sociais Aplicadas, v. 15, n. 1, jan./jun., p. 171-187, 2010.

27. PETERSEN, H. C.; LEWIS, W. C. Managerial Economics. 4. ed. Upper Saddle River (Nova Jersey): Prentice-Hall, 1999.

28. PIMENTA, D. P.; RIBEIRO, K. C. de S. Finanças Comportamentais: Um Estudo sobre a Confiabilidade das Decisões dos Investidores, Analistas e Profissionais de Investimento à Luz da Metodologia Brier Score. In: XIII SEMEAD - SEMINÁRIO EM ADMINISTRAÇÃO - USP, São Paulo, setembro, 2010.

29. PINHEIRO, J. L. (2009). Mercado de capitais: fundamentos e técnicas. 5. ed. São Paulo: Atlas, 2009.

30. RAGSDALE, C. T. Spreadsheet modeling \& decision analysis: a practical introduction to Management Science. 4. ed. Mason (Ohio): South-Western, 2004.

31. ROGERS, P.; SECURATO, J. R.; RIBEIRO, K. C. de S. Finanças Comportamentais no Brasil: Um Estudo Comparativo. Revista de Economia e Administração, v. 6, p. 49-68, 2007.

32. Saaty, L. Decision making with the analytic hierarchy process. Int. J. Services Sciences. 1(1), 83-98, 2008.

33. SHARPE, W.F. A Simplified Model for Portfolio Analysis. Management Science, Baltimore: Institute of Management Sciences, p. 277-293, jan, 1963.

34. SILVA, W. V.; CORSO, J. M. D.; SILVA, S. M. DA.; OLIVEIRA, E. de. Finanças Comportamentais: Análise do perfil comportamental do investidor e do propenso investidor. Revista Eletrônica de Ciência Administrativa (RECADM), v. 7, n. 2, p. 1-14, Novembro, 2008.

35. SPEIDELL, L. S. Investing in the Unknown and the Unknowable - Behavioral Finance in Frontier Markets, The Journal of Behavioral Finance, v. 10, p. 1-8, 2009.

36. SUBRAHMANYAM, A. Behavioural Finance: A Review and Synthesis. European Financial Management, v. 14, n. 1, p. 12-29, 2008.

37. VARIAN, H. R. Microeconomic analysis. 3. ed. Nova York: W. W. Norton \& Company, 1992.

38. YOSHINAGA, C. E.; OLIVEIRA, R. F. DE; SILVEIRA, A. DI M. DA.; BARROS, L. A. B. de C. Finanças Comportamentais: Uma Introdução. Revista de Gestão USP, São Paulo, v. 15, n. 3, p. 25-35, jul/set, 2008. 